\title{
On Tensorial Spaces and BCFW Recursion Relations for Higher Spin Fields
}

\author{
Mirian Tsulaia \\ Center of Elementary Particle Physics, Institute for Theoretical Physics, Ilia State \\ University, Tbilisi 0162, Georgia
}

\begin{abstract}
In this short review we briefly consider two topics in the higher spin gauge theory: the method of "tensorial (super) spaces" and application of BCFW recursion relations to higher spin fields.
\end{abstract}




\section{Contents}

1 Introduction 1

2 Tensorial space 3

2.1 Flat space-time . . . . . . . . . . . . . . . . 3

2.2 AdS space-time . . . . . . . . . . . . . . . . . . 9

3 Cubic and Higher Order Interactions 11

3.1 Cubic vertices on a flat background . . . . . . . . . . . . 11

3.2 A short review of the BCFW method . . . . . . . . . . . 16

3.3 BCFW relations and higher spin fields . . . . . . . . . . 17

\section{Introduction}

Since it has been shown [1]-2] that higher spin gauge theory is a consistent theory of interacting fields with infinitely growing spins, (see [3]-[12] for reviews of different aspects of the subject) this theory is attracting considerable and growing attention.

In the present review we briefly describe two topics in the higher spin gauge theory. In the first part of the review we consider a description of massless higher spin fields in terms of so called "tensorial" (super)spaces. This approach assumes an extension of the original flat or anti de Sitter spaces in terms of extra coordinates. These extra coordinates correspond to spin degrees of freedom. The free field dynamics in this extended space is described in terms of a simple wave equation, which makes the conformal symmetries of the system manifest.

The idea of introducing extra coordinates for the description of spin degrees freedom was first expressed in [13], where it has been conjectured that $\operatorname{OSp}(1 \mid 8)$ group is a symmetry group of full interacting theory of massless higher spin fields in four dimensions. The first explicit realization of this idea was given in [14]-[15] where a model of twistorial superparticle on an extended superspace was considered. In this consideration extra bosonic coordinates were cannonically conjugated to central charges in the corresponding SUSY algebra. The quantization has revealed that the helicity constraint, which is present in an ordinary superparticle model and restricts a superspin of the superparticle to a particular value, does not exist for a tensorial superparticle model. Thus, the spectrum contains an infinite number of higher spin fields. In [16] M. Vasiliev extensively developed this subject, having shown that the first-quantized field equations in tensorial superspace of a bosonic dimension $n(2 n+1)$ and of a fermionic dimension $2 n N$ are $O S p(N \mid 4 n)$ invariant, and for $n=2$ correspond to so called unfolded higher spin field equations in $D=4$. It has also been shown [17] that the theory possesses properties of causality and locality. A detailed analysis of free equations in $D=3,4,6$ and 10 was further given in [18]. One can also consider a tensorial extension of anti de Sitter spaces [19]-[21] and in this way obtain the free unfolded formulation of massless higher spin fields on 
anti de Sitter space-time. Moreover tensorial superspaces are not only an elegant tool for the description of dynamics of free massless higher spin fields on flat and anti de Sitter backgrounds. In [22] (see also [23]-[26]) it has been shown how to describe interactions of higher spin fields with external higher spin currents using this approach.

In the second part of the review we address a problem of consistency for the higher spin theory on the flat space-time background.

An interaction of massless higher spin fields on a flat background, unlike the one on AdS space which naturally bypasses Coleman-Mandula no go theorem is considered to be more problematic. However, a problem of constructing cubic interaction vertices for massless higher spin fields has been extensively studied on the flat space-time background [27]-[30] and interesting solutions for cubic vertices have been found. An important question, however, is if one can build a consistent perturbation theory for massless higher spin fields on Minkowski space-time in order to obtain a nontrivial S-matrix, since the most formidable problems in the higher spin gauge theory on flat background start from four point interactions. One can achieve consistent cubic interactions and closure of the algebra of gauge transformations (even using a finite number of fields) at the level parameter $\times(\text { field })^{2}$. When going to higher order interaction vertices one encounters a requirement to introduce of an infinite number of gauge fields. Moreover, one needs either to introduce quartic interaction vertices or impose some extra conditions on the cubic vertices in order to close the algebra at the level parameter $\times(\text { field })^{3}$. These requirements can in turn lead to a trivial $S$-matrix. Then one needs to continue this procedure to higher (quintic) levels, and so on.

It would be desirable to use the extensive knowledge of cubic vertices on flat space [27]- 30] to obtain information about the consistency of higher order interactions for massless higher spin fields. For this reason one can follow the strategy given in [31]: use the solutions for cubic interaction vertices given in [27, 30] and apply BCFW 32] 34 recursion relations to them. This way one can express four point functions in terms of three point ones, in order to answer the question about the properties (existence) of the corresponding S-matrix. A study of four point vertices and BCFW relations for massless higher spin fields carried out in [31] indicated that consistency of the theory requires an inclusion of extended, and possibly nonlocal, objects* like stringy Pomerons [38] which were used in [39]-[40] to prove BCFW relations for string theory.

The paper is organized as follows:

In Section 2 we review the main properties of tensorial spaces (see [13]-[22], [41][42] for more details $\Phi^{\dagger}$. We consider a tensorial extension of both flat and anti de Sitter spaces and briefly discuss the interaction with higher spin currents in this approach.

*See also [30, 35], 36] for studies of four point functions and the discussion of nonlocalities in the theory and [37. for a general strategy of constructing of higher order vertices.

${ }^{\dagger}$ See also [43] for some mechanical models which lead to higher spin fields 
In Section 3 we briefly review some cubic interaction vertices for massless higher spin fields on the Minkowski background. Then, after a short review of BCFW recursion relations, we apply BCFW recursion relations to theories with cubic interaction vertices described earlier.

\section{Tensorial space}

\subsection{Flat space-time}

Let us formulate the basic idea behind the introduction of tensorial space. We shall mainly concentrate on a tensorial extension of four dimensional $D=4$ Minkowski space-time. A generalization for higher dimensional $D=6$ and $D=10$ spaces will be given later in this Section. Our discussion will include only bosonic tensorial spaces, without their supesymmetric generalizations.

Consider a four dimensional massless scalar field. Its light -like momentum (see for example [44] for a review) $p_{m} p^{m}=0, m=0,1,2,3$ can be parametrized using two commuting mutually complex conjugate Weyl spinors $\lambda_{A}$ and $\bar{\lambda}_{\dot{A}}$ with $A, \dot{A}=1,2$

$$
p^{m}=\lambda^{A}\left(\sigma^{m}\right)_{A \dot{A}} \tilde{\lambda}^{\dot{A}}, \quad \text { or } \quad P_{A \dot{A}}=\lambda_{A} \bar{\lambda}_{\dot{A}}
$$

Obviously since the spinors are commuting, one has $\lambda^{A} \lambda_{A}=\lambda^{\dot{A}} \lambda_{\dot{A}}=0$ and therefore $P^{A \dot{A}} P_{A \dot{A}}=0$. In order to generalize these construction to higher dimensions note that one can equivalently rewrite the equation (2.1) in terms of four dimensional real Majorana spinors $\lambda^{\alpha}$, where $\alpha=1, \ldots, 4$ as

$$
p^{m}=\lambda^{\alpha} \gamma_{\alpha \beta}^{m} \lambda^{\beta}
$$

since due to Fierz identities for Dirac $\left(\gamma^{m}\right)_{\alpha \beta}=\left(\gamma^{m}\right)_{\beta \alpha}$ matrices

$$
\left(\gamma^{m}\right)_{\alpha \beta}\left(\gamma_{m}\right)_{\gamma \delta}+\left(\gamma^{m}\right)_{\alpha \delta}\left(\gamma_{m}\right)_{\beta \gamma}+\left(\gamma^{m}\right)_{\alpha \gamma}\left(\gamma_{m}\right)_{\delta \beta}=0
$$

one has $p^{m} p_{m}=0$. Let us note that since the identity (2.3) is valid not only in four dimensions but in $D=3,4,6,10$, the parametrization (2.2) of a light-like momentum via commuting spinors is valid in these dimensions as well (see [15] for a more detailed discussion on this point).

Let us continue with the four dimensional case. The four momentum $P_{A \dot{A}}$ is cannonically conjugate to coordinates $x^{A \dot{A}}$. One can easily solve the quantum analogue of the equation (2.1)

$$
\left(\frac{\partial}{\partial x^{A \dot{A}}}-i \lambda_{A} \bar{\lambda}_{\dot{A}}\right) \Phi(x, \lambda)=0
$$

to obtain a plane wave solution for the massless scalar particle

$$
\Phi(x, \lambda)=\phi(\lambda) e^{i x^{A \dot{A}} \lambda_{A} \bar{\lambda}_{\dot{A}}}
$$


or in terms of Majorana spinors

$$
\Phi(x, \lambda)=\phi(\lambda) e^{i x_{m} \lambda^{\alpha} \gamma_{\alpha \beta}^{m} \lambda^{\beta}},
$$

with some unknown function $\phi(\lambda)$.

Let us now consider the equation

$$
P_{\alpha \beta}=\lambda_{\alpha} \lambda_{\beta},
$$

which looks like a straightforward generalization of (2.1) and see its implications. A space-time described by the coordinates $X^{\alpha \beta}$ (conjugate to $P_{\alpha \beta}$ ) is now ten dimensional since it is described by a $4 \times 4$ symmetrical matrix. A corresponding basis of symmetrical matrices is represented by four Dirac matrices $\gamma_{\alpha \beta}^{m}$ and six antisymmetric (in $m$ and $n$ ) combinations $\gamma_{\alpha \beta}^{m n}$. Therefore a general expression for the coordinate $X^{\alpha \beta}$ is

$$
X^{\alpha \beta}=\frac{1}{2} x^{m}\left(\gamma_{m}\right)^{\alpha \beta}+\frac{1}{4} y^{m n}\left(\gamma_{m n}\right)^{\alpha \beta} .
$$

The analogue of the wave equation (2.4) is now

$$
\left(\frac{\partial}{\partial x^{\alpha \beta}}-i \lambda_{\alpha} \lambda_{\beta}\right) \Phi(X, \lambda)=0
$$

whereas the solution of the wave equation (2.9) has the form

$$
\Phi(X, \lambda)=e^{i X^{\alpha \beta} \lambda_{\alpha} \lambda_{\beta}} \phi(\lambda) .
$$

At this point one might ask the question: what is the meaning of the equation (2.9) and of the extra coordinates $y^{m n}$ ? The answer to this question is the following: the equation (2.9) is nothing else but Vasiliev's unfolded equations for massless free higher spin fields on four dimensional Minkowski space-time. The wave function $\Phi(X, \lambda)$ depends on both coordinates $x^{m}$ and $y^{m n}$. While $x^{m}$ describes a customary four dimensional Minkowski space time, an expansion of the wave function in terms of powers of $y^{m n}$ generates fields with higher spins on the four dimensional Minkowski space.

In order to demonstrate these statements let us first Fourier transform the wave function (2.10) into a different representation

$$
C(X, Y)=\int d^{4} \lambda e^{-i Y^{\alpha} \lambda_{\alpha}} \Phi(X, \lambda)=\int d^{4} \lambda e^{-i Y^{\alpha} \lambda_{\alpha}+i X^{\alpha \beta} \lambda_{\alpha} \lambda_{\beta}} \phi(\lambda) .
$$

The function $C(X, Y)$ obeys the equation

$$
\left(\frac{\partial}{\partial X^{\alpha \beta}}-i \frac{\partial^{2}}{\partial Y^{\alpha} \partial Y^{\beta}}\right) C(X, Y)=0 .
$$

Further, in order to make the connection with Vasiliev's equations in $D=4$ let us rewrite (2.7) in Weyl notations

$$
P_{A B}=\lambda_{A} \lambda_{B}, \quad \bar{P}_{\dot{A} \dot{B}}=\bar{\lambda}_{\dot{A}} \bar{\lambda}_{\dot{B}}, \quad P_{A \dot{A}}=\lambda_{A} \bar{\lambda}_{\dot{A}}
$$


therefore

$$
\begin{gathered}
\left(\sigma_{A B}^{m n} \frac{\partial}{\partial y^{m n}}+i \frac{\partial^{2}}{\partial Y^{A} \partial Y^{B}}\right) C(x, y, Y)=0 \\
\left(\bar{\sigma}_{\dot{A} \dot{B}}^{m n} \frac{\partial}{\partial y^{m n}}-i \frac{\partial^{2}}{\partial \bar{Y}^{\dot{A}} \partial \bar{Y}^{\dot{B}}}\right) C(x, y, Y)=0
\end{gathered}
$$

and

$$
\left(\sigma_{A \dot{A}}^{m} \frac{\partial}{\partial x^{m}}+i \frac{\partial^{2}}{\partial Y^{A} \partial \bar{Y}^{\dot{A}}}\right) C(x, y, Y)=0 .
$$

Equations (2.14) relate the dependence of $C(x, y, Y)$ on the coordinates $y^{m n}$ to its dependence on $Y^{\alpha}$ and using this relation one can regard the wave function $C\left(x^{m}, Y^{\alpha}\right):=\left.C\left(X^{\alpha \beta}, Y^{\alpha}\right)\right|_{y^{m n}=0}$ at $y^{m n}=0$ as the fundamental field.

The expansion of $C\left(x^{m}, Y\right)$ in terms of $Y^{A}$ and $\bar{Y}^{\dot{A}}$ is

$$
C\left(x^{p}, Y^{A}, \bar{Y}^{\dot{A}}\right)=\sum_{m, n=0}^{\infty} \frac{1}{m ! n !} C_{A_{1} \ldots A_{m}, \dot{B}_{1} \ldots \dot{B}_{n}}\left(x^{p}\right) Y^{A_{1}} \ldots Y^{A_{m}} \bar{Y}^{\dot{B}_{1}} \ldots \bar{Y}^{\dot{B}_{n}}
$$

where reality imposes $\left(C_{A_{1} \ldots A_{m}, \dot{B}_{1} \ldots \dot{B}_{n}}\right)^{*}=C_{B_{1} \ldots B_{n}, \dot{A}_{1} \ldots \dot{A}_{m}}$, and the spin-tensors $C$ are by definition symmetric in the indices $A_{i}$ and in $\dot{B}_{i}$.

The consistency of (2.15) implies the integrability conditions

$$
\frac{\partial^{2}}{\partial Y^{[A} \partial x^{B] \dot{B}}} C\left(x^{C \dot{C}}, Y\right)=0, \quad \frac{\partial^{2}}{\partial \bar{Y}^{[\dot{A}} \partial x^{\dot{B}] B}} C\left(x^{C \dot{C}}, Y\right)=0
$$

Let us recall Vasiliev's unfolded formulation of free higher spin fields in terms of zero-forms. In this formulation the $C_{0,0}$ component (a physical scalar), $C_{A_{1} \ldots A_{2 s}, 0}$ and $C_{0, \dot{A}_{1}, \ldots \dot{A}_{2 s}}$ components of the expansion (2.16) correspond to the physical fields, while the other fields are auxiliary. The latter two fields are the self-dual and antiself-dual components of the spin-s field strength. The nontrivial equations on the dynamical fields are [3] the Klein-Gordon equation for the spin zero scalar field $\partial^{m} \partial_{m} C_{0,0}=0$ and the massless equations for spin $s>0$ field strengths

$$
\partial^{B \dot{B}} C_{B A_{1} \ldots A_{2 s-1}}(x)=0, \quad \partial^{B \dot{B}} C_{\dot{B} \dot{A}_{1} \ldots \dot{A}_{2 s-1}}(x)=0,
$$

which follow from (2.17) $\mathrm{f}$.

\footnotetext{
$\ddagger$ The well known counting of the degrees of freedom is as follows: the symmetric tensor $C_{B A_{1} \ldots A_{2 s-1}}$ has $\left(\begin{array}{c}2 s+1 \\ 2 s\end{array}\right)=2 s+1$ components satisfying $\left(\begin{array}{c}2 s \\ 2 s-1\end{array}\right)=2 s$ independent conditions; this leaves in $C_{B A_{1} \ldots A_{2 s-1}}$ one independent helicity degree of freedom, as is well known for the massless spin $s$ fields in $D=4$. The spin $s$ state of opposite helicity is described by $C_{\dot{B} \dot{A}_{1} \ldots \dot{A}_{2 s-1}}\left(x^{m}\right)$.
} 
All the components of $C\left(x^{m}, Y^{A}, \bar{Y}^{\dot{A}}\right)$ that depend on both $Y^{A}$ and $\bar{Y}^{\dot{A}}$ are auxiliary fields expressed by (2.15) in terms of space-time derivatives of the dynamical fields contained in the analytic fields $C\left(x^{m}, Y^{A}, 0\right)$ and $C\left(x^{m}, 0, Y^{\dot{A}}\right)$ and thus one arrives at the unfolded formulation of 3 .

Let us briefly mention an alternative way to derive the same results. This approach is more convenient for further generalization for $D=6$ and $D=10$. We shall outline the main idea and refer to [18] for the details.

Let us rewrite the equation (2.16) in terms of Majorana spinors $\$$

$$
C(X, Y)=\sum_{n=0}^{\infty} C_{\alpha_{1} \cdots \alpha_{n}}(X) Y^{\alpha_{1}} \cdots Y^{\alpha_{n}}=b(X)+f_{\alpha}(X) Y^{\alpha}+\cdots
$$

and put it into the equation (2.12). Then one finds that all components of $C(X, Y)$ proportional to the higher powers of $Y^{\alpha}$ are expressed in terms of two fields: $b(X)$ and $f_{\alpha}(X)$. As a result of (2.19) these fields satisfy the relations [16]

$$
\begin{aligned}
\partial_{\alpha \beta} \partial_{\gamma \delta} b(X)-\partial_{\alpha \gamma} \partial_{\beta \delta} b(X) & =0 \\
\partial_{\alpha \beta} f_{\gamma}(X)-\partial_{\alpha \gamma} f_{\beta}(X) & =0 .
\end{aligned}
$$

Now the basic objects $b(X)$ and $f_{\alpha}(X)$ depend on both $x^{m}$ and $y^{m n}$. Let us expand these fields in terms of the tensorial coordinates

$$
\begin{aligned}
b(x, y)=\phi(x) & +y^{m_{1} n_{1}} F_{m_{1} n_{1}}(x)+y^{m_{1} n_{1}} y^{m_{2} n_{2}} \hat{R}_{m_{1} n_{1}, m_{2} n_{2}}(x) \\
& +\sum_{s=3}^{\infty} y^{m_{1} n_{1}} \cdots y^{m_{s} n_{s}} \hat{R}_{m_{1} n_{1}, \cdots, m_{s} n_{s}}(x), \\
f^{\alpha}(x, y)= & \psi^{\alpha}(x)+y^{m_{1} n_{1}} \hat{\mathcal{R}}_{m_{1} n_{1}}^{\alpha}(x) \\
& +\sum_{s=\frac{5}{2}}^{\infty} y^{m_{1} n_{1}} \cdots y^{m_{s-\frac{1}{2}} n_{s-\frac{1}{2}}} \hat{\mathcal{R}}_{m_{1} n_{1}, \cdots, m_{s-\frac{1}{2}} n_{s-\frac{1}{2}}}^{\alpha}(x) .
\end{aligned}
$$

Each component field in this expansion is antisymmetric under permutation of indexes $m_{i}$ and $n_{i}$, but is symmetrical with respect to permutation of pairs $\left(m_{i}, n_{i}\right)$ with $\left(m_{j}, n_{j}\right)$. In order to answer the question about the physical meaning of these fields one can use $\gamma$ - matrix identities in four dimensions to rewrite equation (2.20) in an equivalent form

$$
\begin{gathered}
\partial_{p} \partial^{p} b\left(x^{l}, y^{m n}\right)=0, \quad\left(\partial_{p} \partial_{q}-4 \partial_{p r} \partial_{q}^{r}\right) b\left(x^{l}, y^{m n}\right)=0, \quad \epsilon^{p q r t} \partial_{p q} \partial_{r s} b\left(x^{l}, y^{m n}\right)=0 \\
\epsilon^{p q r t} \partial_{q} \partial_{r t} b\left(x^{l}, y^{m n}\right)=0, \quad \partial_{q}{ }^{p} \partial_{p} b\left(x^{l}, y^{m n}\right)=0 .
\end{gathered}
$$

$\S$ As we mentioned before one can of course use either Mayorana or Weyl spinors. The equations written in terms of Majorana spinors are simpler to generalise to higher dimensions, while the equations written in terms of Weyl spinors are simpler to compare with original four dimensional equations of [3]. 
where $\partial_{p}=\frac{\partial}{\partial x^{p}}$ and $\partial_{p q}=\frac{\partial}{\partial y^{p q}}$. The meaning of the equations (2.24) is the following: the first equation is a Klein-Gordon equation. The second equation implies that a trace of the tensor which comes with the $s$ - th power of $y^{m n}$ in the expansion (2.20) is expressed via the second derivative of the tensor which comes with the $s-2$ - th power of $y^{m n}$ in the expansion (2.20). Therefore traces are not independent degrees of freedom and the tensorial fields under consideration are effectively traceless. The third and fourth equations in (2.24) mean that the tensor fields satisfy the Bianchi identities, and the last equation implies that they are co-closed. These are equations for conformal higher spin fields written in terms of curvatures $\hat{\mathcal{R}}_{m_{1} n_{1}, \cdots, m_{s-\frac{1}{2}} n_{s-\frac{1}{2}}}^{\alpha}(x)$. Since in four dimensions equations for higher spin fields are conformally invariant we obtain field equations for all higher spin fields in $D=4$. The treatment of the equation (2.21) which describes half integer higher spin fields in terms of corresponding curvatures is completely analogous to the bosonic one (2.20).

Let us summarize our discussion: in order to describe the dynamics of higher spin fields in four dimensions we have introduced extended ten dimensional space - so called "tensorial space" parametrized by coordinates $X^{\alpha \beta}$ (2.8). The main object is a generating functional for higher spin fields described by $C(X, Y)$ or by $\Phi(X, \lambda)$. the generating functional depends on tensorial coordinates $X^{\alpha \beta}$ and on the commuting spinors $Y^{\alpha}$ or $\lambda^{\alpha}$. The dynamic is encoded into field equations (2.9) or (2.12). To obtain "actual" field equations in terms of only physical coordinates $x^{m}$ one can use two different approaches. In the framework of the first approach one first gets rid of tensorial coordinates $y^{m n}$ and arrives at Vasiliev's unfolded formulation in terms of the functional (2.16). Alternatively, one can first get rid of the commuting spinor variables and arrive at geometric equations formulated in terms of (2.22) and (2.23). In both pictures the equations are formulated in terms of field strength of four dimensional higher spin fields; the difference is that they are represented via either spinorial or vectorial indexes.

Finally, let us give a definition of tensorial spaces in $D=6$ and $D=10$ space-time dimensions. In $D=10$ the twistor-like variable $\lambda_{\alpha}$ is a 16 -component MajoranaWeyl spinor. The gamma-matrices $\gamma_{m}^{\alpha \beta}$ and $\gamma_{m_{1} \cdots m_{5}}^{\alpha \beta}$ form a basis of the symmetric $16 \times 16$ matrices, so the $n=16$ tensorial manifold is parametrized by the coordinates

$$
\begin{gathered}
X^{\alpha \beta}=\frac{1}{16}\left(x^{m} \gamma_{m}^{\alpha \beta}+\frac{1}{2 \cdot 5 !} y^{m_{1} \ldots m_{5}} \gamma_{m_{1} \ldots m_{5}}^{\alpha \beta}\right)=X^{\beta \alpha}, \\
(m=0,1, \ldots, 9 ; \quad \alpha, \beta=1,2, \ldots, 16),
\end{gathered}
$$

where $x^{m}=X^{\alpha \beta} \gamma_{\alpha \beta}^{m}$ are associated with the coordinates of the $D=10$ space-time, while the anti-self-dual coordinates

$$
y^{m_{1} \ldots m_{5}}=X^{\alpha \beta} \gamma_{\alpha \beta}^{m_{1} \ldots m_{5}}=-\frac{1}{5 !} \epsilon^{m_{1} \ldots m_{5} n_{1} \ldots n_{5}} y_{n_{1} \ldots n_{5}},
$$

describe spin degrees of freedom.

The corresponding field equations are again (2.20) and (2.21) and the entire discussion repeats as in the case of $D=4$. The crucial difference is that now the 
expansion (2.22) and (2.23) is performed in terms of the coordinates $y^{m_{1} \ldots m_{5}}$. As a result one obtains a description of conformal fields whose curvatures are self-dual with respect to each set of indexes $\left(m_{1} n_{i} p_{i} q_{i} r_{i}\right)$. These traceless rank $5 s$ tensors $R_{[5]_{1} \ldots[5]_{s}}$ are automatically irreducible under $G L(10, \mathbb{R})$ due to the self-duality property, and are thus associated with the rectangular Young diagrams $(s, s, s, s, s)$ which are made of five rows of equal length $s$ ("multi -five-forms"). The field equations, which are ten-dimensional analogues of four dimensional equations (2.24), can be found in [18].

In $D=6$ the commuting spinor $\lambda_{\alpha}$ is a symplectic Majorana-Weyl spinor. The spinor index can thus be decomposed as $\alpha=a \otimes i(\alpha=1, \ldots, 8 ; a=1,2,3,4$; $i=1,2)$. The tensorial space coordinates $X^{\alpha \beta}=X^{a i b j}$ are decomposed into

$$
\begin{aligned}
& X^{a i b j}=\frac{1}{8} x^{m} \tilde{\gamma}_{m}^{a b} \epsilon^{i j}+\frac{1}{16 \cdot 3 !} y_{I}^{m n p} \tilde{\gamma}_{m n p}^{a b} \tau_{I}^{i j}, \\
& \quad m, n, p=0, \ldots, 5 ; \quad a, b=1, \ldots, 4 ; \quad i, j=1,2 ; \quad I=1,2,3
\end{aligned}
$$

where $\epsilon^{12}=-\epsilon_{12}=1$, and $\tau_{I}^{i j}(I=1,2,3)$ provide a basis of $2 \times 2$ symmetric matrices and are expressed through the usual $S U(2)$ group Pauli matrices, $\tau_{I i j}=\epsilon_{j j^{\prime}} \sigma_{I i}{ }^{j^{\prime}}$. The matrices $\tilde{\gamma}_{m}^{a b}\left(\gamma_{a b}^{m}=1 / 2 \varepsilon_{a b c d} \tilde{\gamma}^{m c d}\right)$ provide a complete set of $4 \times 4$ antisymmetric matrices with upper (lower) indices transforming under an (anti)chiral fundamental representation of the non-compact group $S U^{*}(4) \sim \operatorname{Spin}(1,5)$. For the space of $4 \times 4$ symmetric matrices with upper (lower) indices a basis is provided by the set of self-dual (anti-self-dual) matrices $\left(\tilde{\gamma}^{m n p}\right)^{a b}\left[\gamma_{a b}^{m n p}\right]$,

$$
\left(\tilde{\gamma}^{m n p}\right)^{a b}=\frac{1}{3 !} \epsilon^{m n p q r s} \tilde{\gamma}_{q r s}^{a b}, \quad \gamma_{a b}^{m n p}=-\frac{1}{3 !} \epsilon^{m n p q r s}\left(\gamma_{q r s}\right)_{a b}
$$

The coordinates $x^{m}=x^{a i b j} \gamma_{a b}^{m} \epsilon_{i j}$ are associated with $D=6$ space-time, while the self-dual coordinates

$$
y_{I}^{m n p}=x^{a i b j} \gamma_{a b}^{m n p} \tau_{I i j}=-\frac{1}{3 !} \epsilon^{m n p q r s} y_{q r s}^{I},
$$

describe spinning degrees of freedom.

The discussion repeats again that of the case for $D=4$ and $D=10$. Because of the form of the tensorial coordinates in (2.26) the six dimensional analogue of the expansions (2.22) and (2.23) contains powers of $y_{i}^{m n p}$. Corresponding field strengths, which again describe conformal fields in six dimensions, are self-dual with respect to each set of the indexes $\left(m_{i} n_{i} p_{i}\right)$. In other words, one has an infinite number of conformally invariant (self-dual) "multi-3-form" higher spin fields in the sixdimensional space-time which form the $(2[s]+1)$-dimensional representation of the group $S O(3)$.

It is possible to describe interactions with higher spin currents in terms of tensorial spaces. In order to do so (we shall describe a main idea and refer to [22, [23]-[26] for the detailed discussion and derivations) the equation (2.12) has been generalized 
in [26] to include several commuting variables

$$
\left(\frac{\partial}{\partial X^{\alpha \beta}} \pm i \eta^{i j} \frac{\partial^{2}}{\partial Y^{i \alpha} \partial Y^{j \beta}}\right) C_{ \pm}^{r}(X, Y)=0 .
$$

where $i, j=1, \ldots, r$ and $\eta^{i j}=\eta^{i j}$ is a nondegenerate metric. As we explained above free higher spin fields in $D=4$ are described by the rank one equations in the ten dimensional tensorial space. On the other hand, higher spin currents are fields of rank two $r=2$. These currents obey the equations with off-diagonal $\eta^{i j}[24]$. These currents $J\left(X, Y^{i}\right)$ are bilinear in the higher spin gauge fields $\mathcal{C}_{+}$and $\mathcal{C}_{-}$, which obey rank one equation (2.29) $J=\mathcal{C}_{+} \mathcal{C}_{-}$.

On the other side when considering rank two equations the corresponding tensorial space can be embedded in the higher dimensional tensorial space. From the discussion above it follows that a natural candidate for such higher dimensional space is tensorial extension of $D=6$ space-time. In this way one effectively linearizes the problem since conformal currents in four dimensions are identified with the fields in $D=6[22]$.

\section{$2.2 \quad$ AdS space-time}

A discussion for tensorial extension of $A d S_{4}$ spaces follows the same lines as for tensorial extension of Minkowski space-time [19]-[21]. In order to better explain how a tensorial extension of anti de Sitter space-time looks let us return for a moment to the four dimensional Minkowski space-time and consider the symmetries of the equation (2.7). We shall concentrate mainly on four space-time dimensions. In accordance with the conjecture of [13] the equations (2.7) are invariant under the transformations of the $S p(8)$ group [20]

$$
\delta \lambda_{\alpha}=g_{\alpha}{ }^{\beta} \lambda_{\beta}-k_{\alpha \beta} X^{\beta \gamma}, \quad \delta X^{\alpha \beta}=a^{\alpha \beta}-\left(X^{\gamma \beta} g_{\gamma}{ }^{\alpha}+X^{\gamma \alpha} g_{\gamma}{ }^{\beta}\right)+X^{\alpha \gamma} k_{\gamma \delta} X^{\delta \beta} .
$$

The parameters $a^{\alpha \beta}, g_{\gamma}^{\alpha}$ and $k_{\alpha \beta}$ correspond to the generators $P_{\alpha \beta}$ (translations) $G_{\beta}^{\alpha}\left(G L(4)\right.$ group) and $K_{\alpha \beta}$ (conformal boosts) of the $S p(8)$ group

$$
\begin{aligned}
& {[P, P] }=0, \quad[K, K]=0, \\
& {[P, K] \sim G, \quad[G, G] \sim G, \quad[G, P] \sim P, \quad[G, K] \sim K . }
\end{aligned}
$$

Let us note that under the transformations (2.30) one has

$$
\delta d X^{\alpha \beta}=d X^{\alpha^{\prime} \beta} g_{\alpha^{\prime}}{ }^{\alpha}(X)+d X^{\alpha \beta^{\prime}} g_{\beta^{\prime}}{ }^{\beta}(X),
$$

where $g_{\alpha^{\prime}}^{\alpha}(X)$ are the infinitesimal $S p(8)$ transformations nonlinearly realized on the coset superspace $\frac{S p(8)}{G L(4) \otimes K}$ in terms of $G L(4)$ matrices. We have thus demonstrated

\footnotetext{
『Let us note that $S p(8)$ algebra can be conveniently realized after introducing a twistor variable, conjugate to $\lambda_{\alpha}$ as $\left[\mu^{\alpha}, \lambda_{\beta},\right]=\delta_{\beta}^{\alpha}$. The generators of the $S p(8)$ group will then have a form $P_{\alpha \beta}=\lambda_{\alpha} \lambda_{\beta}, G_{\alpha}^{\beta}=\lambda_{\alpha} \mu^{\beta}, K_{\alpha \beta}=\mu_{\alpha} \mu_{\beta}$.
} 
that the tensorial extension of flat four dimensional Minkowski space is a coset space $\frac{S p(8)}{G L(4) \otimes K}$, and $e^{X_{\alpha \beta} P^{\alpha \beta}}$ is a coset element.

A tensorial extension of $A d S_{4}$ space is just another coset of the $S p(8)$ group. Recall that the usual $A d S_{4}$ space is a coset space $\frac{S O(2,4)}{(S O(1,3) \times D) \otimes K}$ parametrized by the coset element $e^{\mathcal{P}_{m} x^{m}}$. The generators of the $A d S_{4}$ boosts can be singled out from the generators of the four dimensional conformal group $S O(2,4)$ by taking a linear combination of the generators of Poincaré translations $P_{m}$ and conformal boosts $K_{m}$ as $\mathcal{P}_{m}=P_{m}+K_{m}$.

Analogously, for the case of tensorial extension of $A d S_{4}$ space let us consider the generators

$$
\mathcal{P}_{\alpha \beta}=P_{\alpha \beta}+K_{\alpha \beta}, \quad[\mathcal{P}, \mathcal{P}] \sim M, \quad[\mathcal{P}, M] \sim \mathcal{P},
$$

where $M_{\alpha \beta}$ stands for $G_{\{\alpha \beta\}}=G_{\{\beta \alpha\}}$. One can see that the corresponding manifold is an $S p(4)$ group manifold [20] which can be realized as a coset space $\frac{S p(8)}{G L(4) \otimes K}$ with the coset element $e^{(P+K)_{\alpha \beta} X^{\alpha \beta}}$.

The consideration of the corresponding free field equations on tensorial space is simplified by the fact, that vielbiens (Cartan forms) $\omega^{\alpha \beta}=d X^{\mu \nu} \omega_{\mu \nu}^{\alpha \beta}(X)$ on the $S p(2 n)$ group manifold differ from the ones of the flat manifold by $G L(2 n)$ rotations [20]

$$
\omega^{\alpha \beta}(X)=d X^{\alpha^{\prime} \beta^{\prime}} G_{\alpha^{\prime}}{ }^{\alpha}(X) G_{\beta^{\prime}}{ }^{\beta}(X), \quad G_{\beta}^{-1 \alpha}(x)=\delta_{\beta}{ }^{\alpha}+\frac{\varsigma}{4} x_{\beta}{ }^{\alpha},
$$

where the parameter $\varsigma$ can be associated with the inverse of $A d S_{4}$ radius in case of $S p(4)$ group. This property of " $G L(2 n)$ flatness" of $S p(2 n)$ group manifolds, which is a generalization of conformal flatness of usual AdS spaces, can be established by checking that the expression (2.34) solves Maurer-Cartan equations for the $S p(2 n)$ group

$$
d \omega^{\alpha \beta}+\frac{\varsigma}{2} \omega^{\alpha \gamma} \wedge \omega_{\gamma}^{\beta}=0
$$

Further, one has to consider an $S p(4)$ counterpart of the equation (2.9). Recall that for the case of the tensorial extension of Minkowski space one has $P_{\alpha \beta}=i \frac{\partial}{\partial X^{\alpha \beta}}$. Similarly, for the tensorial extension of $A d S_{4}$ one has $\mathcal{P}_{\alpha \beta}=i \nabla_{\alpha \beta}$, where due to the the $G L(4)$ flatness of the $S p(4)$ group manifold one has $\nabla_{\alpha \beta}=G_{\alpha}^{-1 \gamma}(x) G_{\beta}^{-1 \delta}(x) \frac{\partial}{\partial X^{\gamma \delta}}$. The operators $\nabla_{\alpha \beta}$ form $S p(4)$ algebra. Therefore the $S p(4)$ version of the equation (2.9) will have the form

$$
\left[\nabla_{\alpha \beta}-\frac{i}{2}\left(Y_{\alpha} Y_{\beta}+Y_{\beta} Y_{\alpha}\right)\right] \Phi(X, \lambda)=0, \quad Y_{\alpha} \equiv \lambda_{\alpha}+\frac{i \varsigma}{8} \frac{\partial}{\partial \lambda^{\alpha}} .
$$

The reason for the appearance of the terms which are proportional to $\varsigma$ in the equation (2.36) is that these terms ensure that the second term in (2.36) obeys $S p(4)$ algebra, as the derivatives $\nabla_{\alpha \beta}$ do. An $S p(4)$ version of the equation (2.12) now looks like

$$
\left[\nabla_{\alpha \beta}-\frac{i}{2}\left(Y_{\alpha} Y_{\beta}+Y_{\beta} Y_{\alpha}\right)\right] C(X, Y)=0, \quad Y_{\alpha} \equiv i \frac{\partial}{\partial y^{\alpha}}+\frac{\varsigma}{8} y_{\alpha} .
$$


The equations (2.36)-(2.37) can be solved to obtain "plane wave" solutions for tensorial $A d S_{4}$ spaces

$$
\begin{gathered}
\Phi(X, \lambda)=\int d^{4} y \sqrt{\operatorname{det} G^{-1}(x)} e^{i X^{\alpha \beta}\left(\lambda_{\alpha}+\frac{\varsigma}{8} y_{\alpha}\right)\left(\lambda_{\beta}+\frac{\varsigma}{8} y_{\beta}\right)+i \lambda_{\alpha} y^{\alpha}} \varphi(y), \\
C\left(X^{\alpha \beta}, y\right)=\int d^{4} \lambda \sqrt{\operatorname{det} G^{-1}(x)} e^{i x^{\alpha \beta}\left(\lambda_{\alpha}+\frac{\varsigma}{8} y_{\alpha}\right)\left(l_{\beta}+\frac{\varsigma}{8} y_{\beta}\right)-i \lambda_{\alpha} y^{\alpha}} \varphi(\lambda) .
\end{gathered}
$$

Finally, after some algebra one can find equations which are analogous to (2.20)

$$
\begin{gathered}
\nabla_{\alpha[\beta} \nabla_{\gamma] \delta} b(x)=\frac{\varsigma}{16}\left(C_{\alpha[\beta} \nabla_{\gamma] \delta}-C_{\delta[\gamma} \nabla_{\beta] \alpha}+2 C_{\beta \gamma} \nabla_{\alpha \delta}\right) b(x)+ \\
\frac{\varsigma^{2}}{64}\left(2 C_{\alpha \delta} C_{\beta \gamma}-C_{\alpha[\beta} C_{\gamma] \delta}\right) b(x) \\
\nabla_{\alpha[\beta} f_{\gamma]}(x)=-\frac{\varsigma}{4}\left(C_{\alpha[\gamma} f_{\beta]}(x)+2 C_{\beta \gamma} f_{\alpha}(x)\right),
\end{gathered}
$$

where $C_{\alpha \beta}$ is a four-dimensional charge conjugation matrix.

In order to obtain free unfolded equations on $A d S_{4}$ one multiplies the equation (2.37) with $\frac{1}{2} G_{\delta}{ }^{\alpha} \gamma_{m}^{\delta \sigma} G_{\sigma}^{\beta}$ and then takes $y^{m n}=0$ to obtain

$$
\left[\frac{\partial}{\partial x^{m}}+i\left(\frac{1}{4} d x^{m} \omega_{m}^{a b}(x) \gamma_{a b}^{\alpha \beta}+\frac{1}{2} d x^{m} e_{m}^{a}(x) \gamma_{a}^{\alpha \beta}\right) Y_{\alpha} Y_{\beta}\right] C\left(x^{m}, y^{\alpha}\right)=0 .
$$

Let us note that instead of (2.39) , one could have used another form of the generating functional [45], [20], which has been obtained for the conformally flat parametrization of $A d S_{4}$. Again, repeating similar steps as in the Subsection 2.1 for the case of flat Minkowski space-time, one can obtain free unfolded equations for massless higher spin fields on $A d S_{4}[2]$.

\section{Cubic and Higher Order Interactions}

\subsection{Cubic vertices on a flat background}

In this section we briefly recall the BRST method for the construction of cubic interaction vertices on a flat background (a more detailed review as well as generalization to AdS backgrounds can be found in [8], [46]). This method is quite general since it can be used for the construction of nonabelian [27], 33], [47] and abelian [48], [49] interaction vertices. Essentially the method of BRST constructions is nothing else but a method for constructing gauge invariant Lagrangians, where extra gauge degrees of freedom are introduced in order to ensure gauge invariance for unconstrained gauge fields and gauge transformation parameters.

Let us recall that free massless fields with a spin $s$ on a $D$-dimensional flat background satisfy mass-shell and transversality conditions.

$$
\partial^{\mu} \partial_{\mu} \phi_{\mu_{1}, . ., \mu_{s}}(x)=\partial^{\mu_{1}} \phi_{\mu_{1}, . ., \mu_{s}}(x)=0
$$


In case one describes an irreducible higher spin mode one has to add to (3.1) an extra equation $\phi^{\mu_{1}}{ }_{\mu_{1}, \ldots, \mu_{s}}(x)=0$. Without this zero trace condition the equations (3.1) describe fields with spins $s, s-2, \ldots, 1 / 0$ simultaneously.

In order to construct a free Lagrangian which gives (3.1) as a result of equations of motion one can introduce an auxiliary Fock space spanned by oscillators $\left[\alpha_{\mu}, \alpha_{\nu}^{+}\right]=$ $\eta_{\mu \nu}$, with the vacuum defined as $\alpha_{\mu}|0\rangle_{\alpha}=0$. Further, let us introduce operators $l_{0}=-\partial^{\mu} \partial_{\mu}$ (D'Alembertian), $l=-i \alpha^{\mu} \partial_{\mu}$ (divergence) as well as its hermitian conjugate $l^{+}=-i \alpha^{\mu+} \partial_{\mu}$. The BRST charge for this set of operators is very simple since the only nonzero commutator is $\left[l, l^{+}\right]=l_{0}$. Introducing anticommuting ghost variables $c_{0}, c, c^{+}$(with ghost number +1 ), conjugate momenta $b_{0}, b, b^{+}$(with ghost number -1), with anticommutation relations $\left\{c_{0}, b_{0}\right\}=\left\{c, b^{+}\right\}=\left\{c^{+}, b\right\}=1$ as well as ghost vacuum $b_{0}|0\rangle_{g h}=b|0\rangle_{g h}=c|0\rangle_{g h}=0$ and $|0\rangle=|0\rangle_{\alpha} \otimes|0\rangle_{g h}$ one can construct a nilpotent BRST chargell

$$
Q=c_{0} l_{0}+c l^{+}+c^{+} l-c^{+} c b_{0}, \quad Q^{2}=0 .
$$

Solving cohomologies of the BRST charge (3.2) one can obtain conditions (3.1) on the physical field. Because of the presence of ghost variables one has extra degrees of freedom in the system, however the gauge fixing procedure entirely removes these extra degrees of freedom and leads to the equations (3.1) with residual gauge invariance $\delta|\phi\rangle=l^{+}|\lambda\rangle, l_{0}|\lambda\rangle=l|\lambda\rangle=0$

In order to consider cubic interactions one needs to take three copies of the Hilbert space described above. The BRST charge is a sum of "individual" ones $Q=Q_{1}+Q_{2}+Q_{3}$ and is nilpotent since $\left\{Q_{i}, Q_{j}\right\}=0$. A cubic Lagrangian

$$
L=\sum_{i=1,2,3} \int d c_{0}^{i}\left\langle\Phi_{i}\left|Q_{i}\right| \Phi_{i}\right\rangle+g\left(\int d c_{0}^{1} d c_{0}^{2} d c_{0}^{3}\left\langle\Phi_{1}\right|\left\langle\Phi_{2}\right|\left\langle\Phi_{3}|| V\right\rangle+\text { h.c. }\right)+O\left(g^{2}\right)
$$

is invariant up to terms of order $g$ under the transformations

$$
\delta\left|\Phi_{i}\right\rangle=Q_{i}\left|\Lambda_{i}\right\rangle-g \int d c_{0}^{i+1} d c_{0}^{i+2}\left(\left(\left\langle\Phi_{i+1}\right|\left\langle\Lambda_{i+2}\right|+\left\langle\Phi_{i+2}\left|\left\langle\Lambda_{i+1}\right|\right) \mid V\right\rangle\right)+O\left(g^{2}\right)\right.
$$

provided the vertex operator $|V\rangle$ is BRST invariant

$$
Q|V\rangle=0
$$

The procedure of solving of the equation (3.5) is simplified after taking two points into account. From the Lagrangian (3.3) one can conclude that the vertex $|V\rangle$ has the ghost number three, so writing it in the form

$$
|V\rangle=V c_{0}^{1} c_{0}^{1} c_{0}^{1}|0\rangle_{1} \otimes|0\rangle_{2} \otimes|0\rangle_{3} \equiv V|0\rangle_{123}
$$

\footnotetext{
"It is possible to make an AdS deformation of the flat BRST charge [50-51]. The cohomologies of these BRST charges reproduce free field equations on AdS [52].
} 
one can conclude that the function $V$ has the ghost number zero. There are only five Lorentz invariant combinations with ghost number zero: $p^{\mu i} p_{\mu}^{j}, \alpha^{\mu i+} p_{\mu}^{j}, \alpha^{\mu i+} \alpha_{\mu}^{j+}$, $c^{i+} b^{j+}$ and $c^{i+} b_{0}^{j}$; where $p_{\mu}=-i \partial_{\mu}$ and the index $i=1,2,3$ numerates separate Hilbert spaces. Therefore, in general the unknown function $V$ is a series expansion in terms of these combinations with unknown coefficients and the BRST invariance condition (3.5) fixes these coefficients.

The second point is that the number operator $N$, which counts the total number of oscillators $\alpha^{\mu i+}, c^{i+}$ and $b^{i+}$

$$
N=\alpha^{\mu i+} \alpha_{\mu}^{i}+c^{i+} b^{i}+b^{i+} c^{i},
$$

commutes with the BRST charge $Q$. Therefore, the equation (3.5) splits into separate equations according to eigenvalues of the operator (3.7). Finally, one needs to discard BRST trivial vertices, because they correspond to the vertices which can be obtained from the free Lagrangian via field redefinitions. One also has to take into account the vanishing of the total derivative $p_{\mu}^{1}+p_{\mu}^{2}+p_{\mu}^{3}=0$.

After these preliminary remarks we can consider cubic interaction vertices for massless higher spin fields on the flat background in the BRST approach. Let us first consider the solution given in [27] in detail. On the first level i.e., for the vertex with total number of oscillators $N=1$ one has the expansion

$$
\Delta_{1}=Y_{i j} \alpha_{\mu}^{i+} p_{\mu}^{j}+Z_{i j} c^{i+} b_{0}^{j}
$$

on the second level $N=2$

$$
\Delta_{2}=S_{i j} c^{i+} b^{j+}+\frac{P_{i j}}{2} \alpha_{\mu}^{i+} \alpha_{\mu}^{j+}
$$

and for $N=3$

$$
\begin{aligned}
\Delta_{3}= & \tilde{X}_{r s t u}^{(1)}\left(\alpha_{\mu}^{r+} \alpha_{\mu}^{s+}\right)\left(\alpha_{\nu}^{t+} p_{\nu}^{u}\right)+\tilde{X}_{r s t u}^{(2)}\left(c^{r+} b^{s+}\right)\left(\alpha_{\mu}^{t+} p_{\mu}^{u}\right)+ \\
& \tilde{X}_{r s t u}^{(3)}\left(\alpha_{\mu}^{r+} \alpha_{\mu}^{s+}\right)\left(c^{t+} b_{0}^{u}\right)+\tilde{X}_{r s t u}^{(4)}\left(c^{r+} b^{s+}\right)\left(c^{t+} b_{0}^{u}\right) .
\end{aligned}
$$

The coefficients $\tilde{X}_{r s t u}^{(1)}, \tilde{X}_{r s t u}^{(2)}, \tilde{X}_{r s t u}^{(3)}$ and $\tilde{X}_{r s t u}^{(4)}$ obey the symmetry relations

$$
\tilde{X}_{r s t u}^{(1)}=\tilde{X}_{\text {srtu }}^{(1)}, \quad \tilde{X}_{r s t u}^{(3)}=\tilde{X}_{\text {srtu }}^{(3)}, \quad \tilde{X}_{r s t u}^{(5)}=-\tilde{X}_{t s r u}^{(5)} .
$$

Let us note [46] that the expressions for $\Delta_{1}, \Delta_{2}$ and $\Delta_{3}$ do not contain powers of $p^{\mu i} p_{\mu}^{j}$ since this kind of terms would belong to trivial cohomologies of the BRST charge (3.2). The BRST invariance conditions for $\Delta_{1}, \Delta_{2}$ and $\Delta_{3}$ are independent from each other since they have different values of $N$. For (3.8) and (3.9) one gets

$$
\begin{gathered}
Z_{i, i+1}+Z_{i, i+2}=0 \\
Y_{i, i+1}=Y_{i i}-Z_{i i}-1 / 2\left(Z_{i, i+1}-Z_{i, i+2}\right)
\end{gathered}
$$




$$
\begin{gathered}
Y_{i, i+2}=Y_{i i}-Z_{i i}+1 / 2\left(Z_{i, i+1}-Z_{i, i+2}\right) . \\
S_{i j}=P_{i j}=0, \quad i \neq j \\
P_{i i}-S_{i i}=0, \quad i=1,2,3
\end{gathered}
$$

Notice that one can take diagonal elements of $Y_{i j}$ and $Z_{i j}$ equal to zero. Imposing a cyclic symmetry one can see that the solution (3.12) is expressed via a single parameter. Similarly, after imposing cyclic symmetry one can see that the solution (3.13) is expressed via another single parameter. The BRST invariance condition for (3.10) implies

$$
\begin{gathered}
\left(2 \tilde{X}_{r s t u}^{(1)} p_{\mu}^{r} p_{\nu}^{u}-\tilde{X}_{r s t u}^{(2)} p_{\mu}^{s} p_{\nu}^{u}\right) c^{r+} \alpha_{\mu}^{s+} \alpha_{\nu}^{t+}=0, \\
\left(-\tilde{X}_{r s t u}^{(3)} p_{\mu}^{u} p_{\mu}^{u}+\tilde{X}_{r s t u}^{(1)} p_{\mu}^{t} p_{\mu}^{u}\right) c^{t+} \alpha_{\nu}^{r+} \alpha_{\nu}^{s+}=0, \\
\left(-\tilde{X}_{r s t u}^{(5)} p_{\mu}^{u} p_{\mu}^{u}+\tilde{X}_{r s t u}^{(2)} p_{\mu}^{t} p_{\mu}^{u}\right) c^{r+} b^{s+} c^{t+}=0, \\
\left(-\tilde{X}_{r t s u}^{(2)} b_{0}^{t} p_{\mu}^{u}+2 \tilde{X}_{r s t u}^{(3)} b_{0}^{u} p_{\mu}^{r}-\tilde{X}_{r s t u}^{(5)} b_{0}^{u} p_{\mu}^{s}\right) c^{r+} c^{t+} \alpha_{\mu}^{s+}=0, \\
\tilde{X}_{r s t u}^{(5)} b_{0}^{s} c^{s+} c^{r+} c^{t+} b_{0}^{u}=0 .
\end{gathered}
$$

After imposing cyclic symmetry on the coefficients $\tilde{X}_{r s t u}^{(1)}$ under the indexes $r, s, t, u$ these equations can be solved [27] to give the solution given in Table 1 where all entries are proportional to yet another free paramater.

As shown in [27] the solution given in Table 1 reproduces a usual Yang-Mills vertex for three spin one fields provided, the fields are equipped with gauge (Chan -Paton) indexes. However, the solution obtained in [27] has many more implications. In particular, taking an exponential of this solution i.e., considering

$$
\mathcal{V}|0\rangle_{123}=e^{\Delta_{1}+\Delta_{2}+\Delta_{3}}|0\rangle_{123}
$$

results in an of-shell extension of the vertex given in [30] (see also [31, [36] for relevant discussion). Again the dependence of the vertex (3.19) on $p^{\mu i} p_{\mu}^{j}$ can be removed via field redefinitions and the BRST invariance conditions [46].

The solution containing only a part with $\Delta_{1}$ and $\Delta_{2}$ is discussed in detail in [49]. It has been shown that choosing $S_{i i}=1$ makes the theory purely cubic, because four point functions are zero in this case. Therefore the vertex which contains only $\Delta_{1}$ and $\Delta_{2}$ gives trivial interactions beyond the cubic level.

Let us note that cubic vertices on flat space-time might have yet another interesting application. In particular, they can be deformed to obtain interaction vertices on $A d S$ background [53]. A construction of vertices on an AdS background is a very interesting subject in its on right [46]- [47], [53]-[59] and it can also be relevant for studies of AdS/CFT correspondence (see for example [60]-62]) which is, however, beyond the scope of the present review. 


\begin{tabular}{|c|c|c|c|c|} 
Index combination & $\tilde{X}^{(1)}$ & $\tilde{X}^{(2)}$ & $\tilde{X}^{(3)}$ & $\tilde{X}^{(4)}$ \\
1231 & 1 & -2 & -1 & 1 \\
1232 & -1 & 0 & 1 & -1 \\
1233 & 0 & 0 & 0 & 1 \\
1211 & 0 & 0 & 1 & 0 \\
1212 & -1 & 2 & 0 & 0 \\
1213 & -1 & 2 & 0 & 0 \\
1221 & 1 & -2 & 0 & -4 \\
1222 & 0 & 0 & -1 & 1 \\
1223 & 1 & 0 & 0 & -1 \\
1111 & 0 & 0 & 0 & 0 \\
1112 & -1 & -2 & 1 & 0 \\
1113 & 1 & 2 & -1 & 0 \\
1121 & -2 & -4 & 1 & 1 \\
1122 & -6 & -12 & -5 & -4 \\
1123 & 0 & -2 & -1 & -1 \\
1131 & 2 & 4 & -1 & -1 \\
1132 & 0 & 2 & 1 & 1 \\
1133 & 6 & 12 & 5 & 4 \\
2131 & & 0 & & \\
2132 & & 2 & & \\
2133 & & 0 & & \\
2111 & & 0 & & \\
2112 & & 2 & & \\
2113 & & 0 & & \\
2121 & & -2 & & \\
2122 & & 0 & & \\
2123 & & -2 & &
\end{tabular}

Table 1: Empty entries in the table mean that the corresponding value of the coefficient can be recovered from the ones given in the table using the cyclic property of indices (for example $\tilde{X}_{1231}^{(i)}=\tilde{X}_{2312}^{(i)}=\tilde{X}_{3123}^{(i)}$ ) and symmetry properties (3.11) 


\subsection{A short review of the BCFW method}

The key point of BCFW method [32]-[33], which is based upon twistor formulation of gauge theory [63], is that tree level amplitudes constructed using Feynman rules are rational functions of external momenta. Analytic continuation of these momenta on the complex domain turns the amplitudes into meromorphic functions which can be constructed solely by their residues. Since the residues of scattering amplitudes are, due to unitarity, products of lower point on-shell amplitudes the final outcome is a set of powerful recursive relations.

The simplest complex deformation involves only two external momenta

$$
\hat{p}_{i}(z)=p_{i}-q z, \quad \hat{p}_{j}(z)=p_{j}+q z
$$

where $z$ is a complex variable and $q \cdot p_{i}=q \cdot p_{j}=0, q^{2}=0$. In Minkowski space-time this is only possible for complex $q$. As discussed in the Section 2 in four dimensions one can use spinor representations of momenta (2.1). BCFW shift (3.20) corresponds to the following shift in the commuting spinors

$$
\hat{\lambda}_{A}^{(i)}(z)=\lambda_{A}^{(i)}+z \lambda_{A}^{(j)}, \quad \hat{\tilde{\lambda}}_{\dot{A}}^{(j)}(z)=\tilde{\lambda}_{\dot{A}}^{(j)}-z \tilde{\lambda}_{\dot{A}}^{(i)} .
$$

A general amplitude after the BCFW shift becomes a mereomorphic function of a complex variable $z$. Simple poles of the function correspond to the values of $z$ where the propagators of intermediate states go on-shell on the complex plane. Finally, an undeformed amplitude can be computed using Cauchy's theorem

$$
\mathcal{M}_{n}(0)=\frac{1}{2 \pi i} \oint_{z=0} \frac{\mathcal{M}_{n}(z)}{z} d z=-\left\{\sum \operatorname{Res}_{z=\text { finite }}+\operatorname{Res}_{z=\infty}\right\} .
$$

In four dimensions polarization vectors can be also represented in terms of commuting spinors

$$
\begin{aligned}
& \epsilon_{A \dot{A}}^{+}=\frac{\mu_{A} \tilde{\lambda}_{\dot{A}}}{\langle\mu, \lambda\rangle}, \quad \epsilon_{A \dot{A}}^{-}=\frac{\lambda_{A} \tilde{\mu}_{\dot{A}}}{[\tilde{\lambda}, \tilde{\mu}]} \\
& \langle\mu, \lambda\rangle \equiv \mu_{A} \lambda_{B} \epsilon^{A B} \quad[\tilde{\lambda}, \tilde{\mu}] \equiv \tilde{\mu}_{\dot{A}} \tilde{\lambda}_{\dot{B}} \epsilon^{\dot{A} \dot{B}}
\end{aligned}
$$

with $\mu_{A}$ and $\tilde{\mu}_{\dot{A}}$ arbitrary reference spinors. Polarizations of higher spin states are given by products of the polarizations for spin one

$$
\epsilon_{A_{1} \dot{A}_{1} \ldots A_{s} \dot{A}_{s}}^{+}=\prod_{i=1}^{s} \epsilon_{A_{i} \dot{A}_{i}}^{+} \quad \epsilon_{A_{1} \dot{A}_{1} \ldots A_{s} \dot{A}_{s}}^{-}=\prod_{i=1}^{s} \epsilon_{A_{i} \dot{A}_{i}}^{-} .
$$

In order for the BCFW recursion relation to be applicable the deformed amplitude should vanish when $z \rightarrow \infty$. This is because a pole at infinity, unlike the ones for finite values of $z$, does not have an interpretation as product of lower point amplitudes. In [34] a very interesting criterion was derived in order to classify, in 
four dimensions, which theories are constructible (have zero pole at infinity) under BCFW deformations. The criterion has been stated explicitly for the four-point function and it is a necessary condition for a theory to have zero residue at infinity. Say we denote by $\mathcal{M}^{(i, j)}(z)$ the four-point function under deformation of particles $i$ and $j$. Assume further that the helicities $h_{2}$ and $h_{4}$ are negative while $h_{1}$ is positive. The criterion advocates that

$$
\mathcal{M}_{4}^{(1,2)}(0)=\mathcal{M}_{4}^{(1,4)}(0)
$$

Note that in $M^{(1,2)}(z)$ only poles from the $t, u$ channels on the complex z-plane will contribute since the expression $1 /\left(p_{1}(z)+p_{2}(z)\right)^{2}=1 /\left(p_{1}+p_{2}\right)^{2}$ is $z$-independent and it does not give a pole. Similarly for $M^{(1,4)}(0)$ only poles from the $s, u$ channels on the complex-z plane will contribute.** So the crossing symmetry condition (3.25) is a highly nontrivial constraint.

\subsection{BCFW relations and higher spin fields}

BCFW recursion relations have wide applications in Yang-Mills gauge theories and supergavities (see [64] for a review), whereas the application of BCFW relations to string theory have been discussed relatively recently [39] - [40], 65]-[66] (see also [67] 69. for interactions between higher spin fields in string theory). The main ingredient in this consideration is the Pomeron vertex operator introduced in [38]. This vertex operator appears when considering the Operator Product Expansion(OPE) between string vertex operators in a Regge regime $s \gg t$ (a high energy limit). The Pomeron vertex operator can be obtained from OPE for usual string vertex operators in a Regge regime $s \gg t$

$$
\mathcal{V}_{P} \sim C_{n} \Gamma\left(\alpha^{\prime} p^{2}-1\right) e^{i p \cdot X}[q \cdot \partial X]^{1-\alpha^{\prime} p^{2}}
$$

where $p=p_{1}+p_{2}$ and $q=p_{1}-p_{2}$. The operator $C_{n}$ depends on the states for which we consider OPE and is a function of their polarizations and momenta. Pomerons are physical composite states, but with a fractional oscillator number, and therefore they are outside a normal Hilbert space

$$
L_{0} \mathcal{V}_{P}=0, \quad L_{1} \mathcal{V}_{P}=0
$$

Taking into account Pomeron vertex operators when considering four point functions, say for a bosonic string, one can show that the typical behavior under BCFW shift is

$$
\mathcal{M}(z) \sim z^{n+1+\alpha^{\prime} P_{12}^{2}}
$$

i.e., open bosonic string amplitudes are constructible in the regime $n+1+\alpha^{\prime} P_{12}^{2}<$ 0 . The inclusion of the Pomeron vertex operator when considering BCFW shift is

\footnotetext{
${ }^{* *}$ We use the following definition for Mandelstam variables $s=\left(p_{1}+p_{2}\right)^{2}, t=\left(p_{1}+p_{4}\right)^{2}, u=$ $\left(p_{1}+p_{3}\right)^{2}$.
} 
natural. Indeed the Pomeron vertex operator appears at the Regge (high energy) limit of the bosonic string and, on the other hand, BCFW shift of the momenta gives as high energy limit as we take $z \rightarrow \infty$.

Now one can try to apply similar arguments for massless higher spin theories. Below we give only some brief arguments and refer to [31] for a detailed discussion. Let us recall that free field equations can be recovered from the usual BRST charge for the bosonic string by taking $\alpha^{\prime} \rightarrow \infty$ limit (see, for example, [51]) and one can further consider interactions in this limit [30]. Therefore, when considering BCFW relations for these theories it is natural to also include Pomeron vertices in the theory (which are relevant to the tensile bosonic string theory in the high energy limit) and which make the theory constructible. Let us also note that in the high energy limit the Pomeron vertex satisfies the physical state conditions.

As a spin of intermediate fields grows a dimension of the coupling constant becomes smaller and relevant diagrams become more divergent. Therefore, the simplest system which includes a coupling of a massless higher spin field with two scalar fields contains a lot of information i.e, it allows us to indicate a problem. Below we give two explicit examples for four point functions, where external particles are scalars which exchange higher spin fields i.e., consider an analogy of Veneziano amplitude. We consider charged scalars since this allows a coupling to odd spins as well. A coupling of an irreducible higher spin mode with two scalars is described by the cubic vertex 70

$$
\mathcal{L}_{\text {int }}^{00 s}=\kappa^{1-h} N_{h} \frac{\Psi_{h}^{\mu_{1} \ldots \mu_{h}} J_{h ; \mu_{1} \ldots \mu_{h}}^{1 ; 2}}{h !}+\text { h.c. }
$$

where

$$
J_{h ; \mu_{1} \ldots \mu_{h}}^{1 ; 2}=\sum_{r=0}^{h}\left(\begin{array}{c}
h \\
r
\end{array}\right)(-1)^{r}\left(\partial^{\mu_{1}} \ldots \partial^{\mu_{r}} \phi_{1}\right)\left(\partial^{\mu_{r+1}} \ldots \partial^{\mu_{h}} \phi_{2}\right)
$$

The consideration of only cubic interactions does not determine the constants $N_{h}$. We examine two different possibilities $N_{h}=h$ ! - "field theory" coupling, when the coupling does not depend on spin and $N_{h}=\sqrt{h !}$ - "string theory" coupling. The form of the latter coupling can be deduced from the vertices considered in the previous subsection and they correspond to the fields belonging to leading Regge trajectories. One can also consider reducible higher spin modes and decompose them into irreducible ones following [71].

The corresponding four point functions can be computed in two different ways: either using the standard technique of Feynman diagrams, or using spinorial representations for helicities described earlier in this Section. In particular, one can show 
that $\mathcal{M}_{4}^{(1,2)}(0)$ is given by the expression [34]

$$
\begin{aligned}
\mathcal{M}_{4}^{(1,2)}(0)= & \sum_{h>\max \left(-\left(h_{1}+h_{4}\right),\left(h_{2}+h_{3}\right)\right)}\left(\kappa_{1-h_{1}-h_{4}-h}^{A} \kappa_{1+h_{2}+h_{3}-h}^{H} \frac{\left(-P_{3,4}^{2}\right)^{h}}{P_{1,4}^{2}}\left(\frac{[1,4][3,4]}{[1,3]}\right)^{h_{4}}\right. \\
& \left.\left(\frac{[1,3][1,4]}{[3,4]}\right)^{h_{1}}\left(\frac{\langle 3,4\rangle}{\langle 2,3\rangle\langle 2,4\rangle}\right)^{h_{2}}\left(\frac{\langle 2,4\rangle}{\langle 2,3\rangle\langle 3,4\rangle}\right)^{h_{3}}\right) \\
& +\sum_{h>\max \left(-\left(h_{1}+h_{3}\right),\left(h_{2}+h_{4}\right)\right)}(4 \leftrightarrow 3) .
\end{aligned}
$$

where $P_{i, j}=p_{i}+p_{j}$ and coupling constant $k_{H}\left(k_{A}\right)$ is required to give the right dimension to the part of the amplitude which is holomorphic (antiholomorphic) in the spinor variables $\lambda^{(i)}\left(\tilde{\lambda}^{(i)}\right)$. The subscript of the coupling constants denotes their mass dimension. The amplitude $\mathcal{M}_{4}^{(1,2)}(0)$ can be obtained from (3.31) by simply exchanging labels 2 and 4 .

Now let us apply the formula (3.31) to the tree-level scattering process for two scalars with the same charge $\phi\left(p_{1}\right) \phi\left(p_{2}\right) \rightarrow \phi\left(-p_{3}\right) \phi\left(-p_{4}\right)$. Considering first the "field theory" coupling i.e., taking $N_{h}=h$ ! in (3.29) one obtains for coupling constants $k_{H}=k_{A}=\kappa^{1-h}$ and therefore

$$
\begin{gathered}
\mathcal{M}_{4}^{(1,2)}(0)=\sum_{h \in \mathbb{Z}} \kappa^{2-2 h}\left(-P_{3,4}^{2}\right)^{h}\left(\frac{1}{P_{1,4}^{2}}+\frac{1}{P_{1,3}^{2}}\right), \\
\mathcal{M}_{4}^{(1,4)}(0)=\sum_{h \in \mathbb{Z}} \kappa^{2-2 h}\left(-P_{1,4}^{2}\right)^{h} \frac{1}{P_{1,3}^{2}},
\end{gathered}
$$

or in terms of the Madelstam variables

$$
\mathcal{M}_{4}^{(1,2)}(0)=\kappa^{2} \frac{1}{1+\kappa^{-2} s}\left(\frac{-s}{t u}\right), \quad \mathcal{M}_{4}^{(1,4)}(0)=\kappa^{2} \frac{1}{1+\kappa^{-2} t}\left(\frac{1}{u}\right) .
$$

Let us note that in the expression for $\mathcal{M}_{4}^{(1,4)}(0)$ the s-channel amplitude is absent due to the charge conservation. It is useful to compute the same amplitude using standard Feynman diagram techniques [72] for this specific choice of coupling constants. Doing so one obtains in t-channel:

$$
\mathcal{M}_{4}^{t} \sim \frac{\kappa^{2}}{t}\left(\frac{1}{1+\frac{\kappa^{-2}}{4}(\sqrt{s}+\sqrt{-u})^{2}}+\frac{1}{1+\frac{\kappa^{-2}}{4}(\sqrt{s}-\sqrt{-u})^{2}}-1\right) .
$$

In a similar way one can compute the u-channel amplitude and add it to (3.34) in order to compare to $\mathcal{M}_{4}^{(1,2)}(0)$ given in (3.33). The amplitudes (3.34) vanish at the complex infinity after making a BCFW shift and taking $z \rightarrow \infty$ which means that the theory should be constructable. Obviously four point amplitudes (3.33) do not pass the four particle test (3.25). This means in turn that either the theory must have either a trivial S-matrix or there is a pathology in its definition. 
As one can see from (3.34) there is a pole which depends on kinematic variables i.e., there should be some extended object in the theory. The BCFW computation tells us the same thing through its failure of crossing symmetry under BCFW deformations, which suggests that at some finite distance on the complex kinematic variables plane the massless theory has some ingredient missing in its definition. Performing the BCFW shift in (3.34) for the particles 1 and 2 one can easily verify that there are three poles on the complex z-plane for the t-channel contribution and three for the $\mathrm{u}$-channel, respectively. The one which comes from the massless t-pole of (3.34) is the one whose $\operatorname{Res}(\mathcal{M}(z) / z)$ reproduces the t-channel pole of (3.32). On this pole the amplitude factorizes into two scalar three point amplitudes which are, however, dressed with some form factors. The other two poles of (3.34) should come from the "extended object" poles which contribute the form factors of (3.33). To get the u-channel pole of (3.32) we will need to compute the residues of the u-channel amplitude $\mathcal{M}_{4}^{u}$ in a similar manner.

Now one can repeat the same arguments for the string theory coupling. In particular, one can see that the crossing symmetry condition (3.25) fails again since four point functions computed with the help of (3.31)

$$
\mathcal{M}_{4}^{(1,2)}(0)=e^{-\kappa^{-2} s}\left(\frac{-s}{t u}\right), \quad \mathcal{M}_{4}^{(1,4)}(0)=e^{-\kappa^{-2} t}\left(\frac{1}{u}\right)
$$

are not equal to each other. A similar conclusion can be made either by directly evaluating four point functions using Feynman diagram techniques or constructing quartic vertices using the BRST approach [31]. In the latter approach one introduces four Hilbert spaces and has therefore BRST charges $Q_{M}, M=1,2,3,4$. Considering for simplicity a part of the cubic vertex (3.19) which contains only $\Delta_{1}$ one arrives to the equation for the quartic vertex

$$
\left(Q_{1}+Q_{2}+Q_{3}+Q_{4}\right)\left|V_{4}\right\rangle=18\left(Z_{a^{\prime} 1} c^{+a^{\prime}}+Z_{a 1} c^{+a}\right) e^{Y_{1 i^{\prime}} Y_{1 i} p_{\mu}^{i^{\prime}} p_{\mu}^{i}} e^{M} c_{0}^{2} c_{0}^{3} c_{0}^{2^{\prime}} c_{0}^{3^{\prime}}|0\rangle_{232^{\prime} 3^{\prime}}
$$

where

$$
M=Y_{a i} \alpha_{\mu}^{+a} p_{\mu}^{i}+Y_{a^{\prime} i^{\prime}} \alpha_{\mu}^{+a^{\prime}} p_{\mu}^{i^{\prime}}+Z_{a b} c^{+a} b_{0}^{b}+Z_{a^{\prime} b^{\prime}} c^{+a^{\prime}} b_{0}^{b^{\prime}}
$$

with $i / i^{\prime}=1,2,3 / 1,2^{\prime}, 3^{\prime}$, and $a, b / a^{\prime} b^{\prime}=2,3 / 2^{\prime} 3^{\prime}$. The equation (3.37) can be solved to give

$$
\left|V_{4}\right\rangle=F(p) e^{M} c_{0}^{2} c_{0}^{3} c_{0}^{2^{\prime}} c_{0}^{3^{\prime}}|0\rangle_{232^{\prime} 3^{\prime}}, \quad F(p)=18 \frac{e^{Y_{1 i^{\prime}} Y_{1 i} p_{\mu}^{i^{\prime}} p_{\mu}^{i}}}{\left(p_{\mu}^{2}+p_{\mu}^{3}\right)\left(p_{\mu}^{2^{\prime}}+p_{\mu}^{3^{\prime}}\right)}
$$

Obviously the full solution is given by acting with the above given vertex on all non-cyclic permutations of the external states $\mathcal{L}_{4} \sim\left\langle 1,2,3,4 \mid V_{4}\right\rangle_{s}+\left\langle 1,3,2,4 \mid V_{4}\right\rangle_{u}+$ $\left\langle 1,4,2,3 \mid V_{4}\right\rangle_{t}$ where each contribution has subscript indicating the massless pole on the corresponding kinematic variable which comes from the definition of $F(p)$ in (3.38). 
Computing the four point function with the Feynman rules and using the interaction vertices given above

$$
\mathcal{M}_{4}^{t} \sim-\frac{\kappa^{2}}{t} \exp \left(-\kappa^{-2} s\right) .
$$

On the other side computing the four point function for tachyons using the Pomeron vertex operator in the bosonic string and taking the naive $\alpha^{\prime} \rightarrow \infty$ limit gives

$$
\mathcal{M}_{4}^{t} \sim \frac{1}{\sqrt{t}}\left(\frac{t}{s}\right)^{\kappa^{-2} t-1} e^{-\kappa^{-2} t}
$$

where $\kappa$ is the critical string length we have assumed in order for our formulas to make sense. This should be compared to (3.39) where we see the marked difference between the two expressions: for the region $s \gg t$ where the two expressions apply one goes as $e^{-\kappa^{-2} s}$ and the other one has the typical Pomeron behavior $s^{\kappa^{-2} t-1}$. Our discussion suggests that massless current exchanges alone cannot lead to the above behavior and one can assume that the expression in (3.40) is the appropriate asymptotic behavior of the tensionless limit of the four point string amplitude for BCFW deformations $s \sim-u \sim q z$.

Therefore the analysis of the examples above suggests that interacting higher spin theories in flat background with only point particle states although not improbable, might not be the ones related to the high energy limit of string theory. Although one can add perturbatively quartic and higher vertices in the Lagrangian, consistently with gauge invariance at each order, a non-trivial S-matrix, its analyticity properties and BCFW constructibility seem to imply that: one should consider higher spin theories in a larger frame which includes extended and/or non-local objects in their spectrum.

Acknowledgments. I am grateful to I. Bandos, X. Bekaert, A. Fotopoulos and D. Sorokin for numerous discussions and collaboration on the topics presented in the review. I would like especially thank A. Fotopoulos for reading the manuscript and for his comments. I am grateful to my family who turned my stay in Auckland, New Zealand into a wonderful experience.

\section{References}

[1] E. S. Fradkin and M. A. Vasiliev, Nucl. Phys. B 291, 141 (1987).

[2] M. A. Vasiliev, Phys. Lett. B 243, 378 (1990), M. A. Vasiliev, Phys. Lett. B 285, 225 (1992), M. A. Vasiliev, Phys. Lett. B 567, 139 (2003) arXiv:hep-th/0304049]. 
[3] M. A. Vasiliev, hep-th/9910096.

M. A. Vasiliev, Fortsch. Phys. 52, 702 (2004) arXiv:hep-th/0401177].

[4] D. Sorokin, AIP Conf. Proc. 767, 172 (2005) arXiv:hep-th/0405069].

[5] N. Bouatta, G. Compere and A. Sagnotti, arXiv:hep-th/0409068.

[6] I. A. Bandos, AIP Conf. Proc. 767, 141 (2005) hep-th/0501115.

[7] X. Bekaert, S. Cnockaert, C. Iazeolla and M. A. Vasiliev, arXiv:hep-th/0503128.

[8] A. Fotopoulos and M. Tsulaia, Int. J. Mod. Phys. A 24, 1 (2009) arXiv:0805.1346 [hep-th]].

[9] A. Campoleoni, Riv. Nuovo Cim. 033, 123 (2010) arXiv:0910.3155 [hep-th]].

[10] D. Francia, J. Phys. Conf. Ser. 222, 012002 (2010) [arXiv:1001.3854 [hep-th]].

[11] X. Bekaert, N. Boulanger and P. Sundell, arXiv:1007.0435 [hep-th].

[12] A. Sagnotti, arXiv:1112.4285 [hep-th].

[13] C. Fronsdal, In *Fronsdal, C. ( Ed.): Essays On Supersymmetry*, 163-265 and Calif. Univ. Los Angeles - UCLA-85-TEP-10 (85,REC.JUN.) 111 P. (508632)

[14] I. A. Bandos and J. Lukierski, Mod. Phys. Lett. A 14, 1257 (1999) hep-th/9811022.

[15] I. A. Bandos, J. Lukierski and D. P. Sorokin, Phys. Rev. D 61, 045002 (2000) hep-th/9904109.

[16] M. A. Vasiliev, Phys. Rev. D 66, 066006 (2002) hep-th/0106149.

[17] M. A. Vasiliev, hep-th/0111119.

[18] I. Bandos, X. Bekaert, J. A. de Azcarraga, D. Sorokin and M. Tsulaia, JHEP 0505, 031 (2005) hep-th/0501113.

[19] V. E. Didenko and M. A. Vasiliev, J. Math. Phys. 45, 197 (2004) hep-th/0301054.

[20] M. Plyushchay, D. Sorokin and M. Tsulaia, JHEP 0304, 013 (2003) hep-th/0301067.

[21] M. Plyushchay, D. Sorokin and M. Tsulaia, hep-th/0310297.

[22] O. A. Gelfond and M. A. Vasiliev, arXiv:1012.3143 [hep-th].

[23] M. A. Vasiliev, arXiv:0707.1085 [hep-th]]. 
[24] O. A. Gelfond and M. A. Vasiliev, JHEP 0903, 125 (2009) arXiv:0801.2191 [hep-th]].

[25] O. A. Gelfond and M. A. Vasiliev, JHEP 0912, 021 (2009) arXiv:0901.2176 [hep-th]].

[26] O. A. Gelfond and M. A. Vasiliev, Theor. Math. Phys. 145, 1400 (2005) [Teor. Mat. Fiz. 145, 35 (2005)] hep-th/0304020].

[27] A. K. H. Bengtsson, Class. Quant. Grav. 5, 437 (1988).

[28] R. R. Metsaev, arXiv:0712.3526 [hep-th]], R. R. Metsaev, Nucl. Phys. B 759, 147 (2006) arXiv:hep-th/0512342].

[29] R. Manvelyan, K. Mkrtchyan and W. Ruehl, Nucl. Phys. B 836, 204 (2010) arXiv:1003.2877,

R. Manvelyan, K. Mkrtchyan and W. Ruehl, arXiv:1002.1358.

[30] A. Sagnotti and M. Taronna, Nucl. Phys. B 842, 299 (2011) arXiv:1006.5242 [hep-th]].

[31] A. Fotopoulos and M. Tsulaia, JHEP 1011, 086 (2010) arXiv:1009.0727 [hepth]].

[32] R. Britto, F. Cachazo and B. Feng, Nucl. Phys. B 715, 499 (2005) arXiv:hep-th/0412308.

[33] R. Britto, F. Cachazo, B. Feng and E. Witten, Phys. Rev. Lett. 94 (2005) 181602 arXiv:hep-th/0501052.

[34] P. Benincasa and F. Cachazo, arXiv:0705.4305 [hep-th].

[35] D. Polyakov, Phys. Rev. D 83, 046005 (2011) [arXiv:1011.0353 [hep-th]].

[36] M. Taronna, arXiv:1107.5843 [hep-th].

[37] A. K. H. Bengtsson, J. Math. Phys. 48 (2007) 072302 arXiv:hep-th/0611067.

[38] R. C. Brower, J. Polchinski, M. J. Strassler and C. I. Tan, JHEP 0712 (2007) 005 arXiv:hep-th/0603115.

[39] C. Cheung, D. O'Connell and B. Wecht, JHEP 1009, 052 (2010) arXiv:1002.4674 [hep-th]].

[40] R. H. Boels, D. Marmiroli and N. A. Obers, JHEP 1010, 034 (2010) arXiv:1002.5029 [hep-th]].

[41] I. Bandos, P. Pasti, D. Sorokin and M. Tonin, JHEP 0411, 023 (2004) hep-th/0407180. 
[42] E. Ivanov and J. Lukierski, Phys. Lett. B 624, 304 (2005) hep-th/0505216. S. Fedoruk, E. Ivanov and J. Lukierski, Phys. Lett. B 641, 226 (2006) hep-th/0606053.

[43] F. Bastianelli, O. Corradini and E. Latini, JHEP 0811, 054 (2008) arXiv:0810.0188 [hep-th]].

O. Corradini, JHEP 1009, 113 (2010) [arXiv:1006.4452 [hep-th]].

[44] F. Cachazo and P. Svrcek, PoS RTN2005 (2005) 004 arXiv:hep-th/0504194.

[45] K. I. Bolotin and M. A. Vasiliev, Phys. Lett. B 479, 421 (2000) [hep-th/0001031].

[46] I. L. Buchbinder, A. Fotopoulos, A. C. Petkou and M. Tsulaia, Phys. Rev. D 74 (2006) 105018 arXiv:hep-th/0609082].

[47] A. Fotopoulos, N. Irges, A. C. Petkou and M. Tsulaia, JHEP 0710 (2007) 021 arXiv:0708.1399 [hep-th]].

A. Fotopoulos and M. Tsulaia, arXiv:1007.0747 [hep-th].

[48] I. G. Koh and S. Ouvry, Phys. Lett. B 179, 115 (1986) [Erratum-ibid. 183B, $434(1987)]$.

[49] A. Fotopoulos and M. Tsulaia, Phys. Rev. D 76, 025014 (2007) arXiv:0705.2939 [hep-th]].

[50] I. L. Buchbinder, A. Pashnev and M. Tsulaia, Phys. Lett. B 523 (2001) 338 arXiv:hep-th/0109067.

I. L. Buchbinder, A. Pashnev and M. Tsulaia, hep-th/0206026.

I. L. Buchbinder, V. A. Krykhtin and A. A. Reshetnyak, [arXiv:hep-th/0703049].

I. L. Buchbinder, V. A. Krykhtin and P. M. Lavrov, Nucl. Phys. B 762 (2007) 344 arXiv:hep-th/0608005.

K. B. Alkalaev and M. Grigoriev, Nucl. Phys. B 835, 197 (2010) arXiv:0910.2690].

C. Burdik and A. Reshetnyak, arXiv:1111.5516 [hep-th].

[51] A. Sagnotti and M. Tsulaia, Nucl. Phys. B 682 (2004) 83 arXiv:hep-th/0311257.

[52] R. R. Metsaev, [arXiv:hep-th/9810231],

R. R. Metsaev, Phys. Lett. B 419, 49 (1998) [arXiv:hep-th/9802097].

[53] M. Vasiliev, arXiv:1108.5921 [hep-th].

[54] K. B. Alkalaev and M. A. Vasiliev, Nucl. Phys. B 655, 57 (2003) hep-th/0206068. 
[55] D. Francia, J. Mourad and A. Sagnotti, Nucl. Phys. B 773, 203 (2007) arXiv:hep-th/0701163].

D. Francia, J. Mourad and A. Sagnotti, Nucl. Phys. B 804, 383 (2008) arXiv:0803.3832 [hep-th]].

[56] D. Polyakov, Phys. Rev. D 84, 126004 (2011) [arXiv:1106.1558 [hep-th]].

[57] N. Boulanger, E. D. Skvortsov and Y. .M. Zinoviev, J. Phys. A A 44, 415403 (2011) arXiv:1107.1872 [hep-th]].

[58] Y. .M. Zinoviev, Class. Quant. Grav. 29, 015013 (2012) arXiv:1107.3222 [hepth]].

[59] E. Joung and M. Taronna, arXiv:1110.5918 [hep-th].

[60] R. d. M. Koch, A. Jevicki, K. Jin and J. P. Rodrigues, Phys. Rev. D 83, 025006 (2011) arXiv:1008.0633 [hep-th]].

[61] M. Henneaux and S. -J. Rey, JHEP 1012, 007 (2010) arXiv:1008.4579 [hep-th]].

[62] A. Campoleoni, S. Fredenhagen, S. Pfenninger and S. Theisen, JHEP 1011, 007 (2010) arXiv:1008.4744 [hep-th]].

[63] E. Witten, Commun. Math. Phys. 252 (2004) 189 arXiv:hep-th/0312171.

[64] B. Feng and M. Luo, arXiv:1111.5759 [hep-th].

[65] A. Fotopoulos and N. Prezas, Nucl. Phys. B 845, 340 (2011) arXiv:1009.3903 [hep-th]].

[66] A. Fotopoulos, J. Phys. A 44, 235402 (2011) [arXiv:1010.6265 [hep-th]].

[67] O. Schlotterer, Nucl. Phys. B 849, 433 (2011) [arXiv:1011.1235 [hep-th]].

[68] W. -Z. Feng and T. R. Taylor, Nucl. Phys. B 856, 247 (2012) arXiv:1110.1087 [hep-th]].

[69] R. H. Boels, arXiv:1201.2655 [hep-th].

[70] F. A. Berends, G. J. H. Burgers and H. van Dam, Nucl. Phys. B 271, 429 (1986),

[71] A. Fotopoulos and M. Tsulaia, JHEP 0910, 050 (2009) arXiv:0907.4061 [hepth]].

[72] X. Bekaert, E. Joung and J. Mourad, JHEP 0905, 126 (2009) arXiv:0903.3338 [hep-th]]. 